\title{
A 45 Year Old Woman With Progressive Dyspnea and Multiple Venous Thromboses
}

\author{
$\underline{\text { Eduardo B. Saad }}^{*}, \underline{\text { Carlos Eduardo Reis }}^{\underline{Y}}, \underline{\text { Cynthia F. Pontes }}^{\underline{Y}}$, M.D. \\ Ricardo Amorin ${ }^{Y}$, M.D.
}

To whom correspondence should be addressed:

*Instituto de Doenças do Tórax, Caixa Postal 68019, Cep 21944-970, Cidade Universitária, Rio de Janeiro, R.J., Brasil

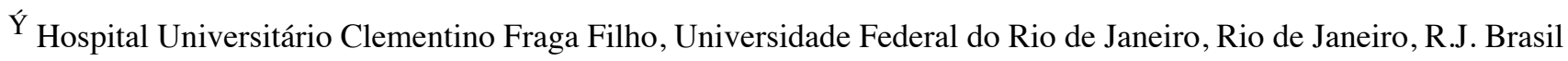

§ Clínica São Vicente de Gávea, Rio de Janeiro, R.J., Brasil

\section{THE CASE}

A 45 year old woman was admitted to the Federal University of Rio de Janeiro Hospital (Rio de Janeiro, Brazil) with acute onset of dyspnea at rest, which started three days prior to admission, and was worsened by minimal effort. This was accompanied by left posterior chest pain that worsened on deep inspiration.

The patient has a two year history of dyspnea on moderate activity accompanied by a dry cough, sometimes productive of bloody sputum, fatigue, bilateral leg edema, and increased abdominal girth. She also complained of dyspnea when lying down, multiple migrating small painful vessels throughout the thorax and abdomen, and an undetermined amount of weight loss. Thereafter, symptoms progressively worsened until dyspnea on minimal effort was present and the patient became essentially bedridden.

One year before admission, thrombosis of the inferior vena cava and multiple deep venous thromboses of both legs were diagnosed, and the patient was started on warfarin. Three months prior to admission, she had thrombosis of the right subclavian, axillary and brachial veins and partial thrombosis of the right internal jugular vein. At this time, a hematological investigation for a procoagulant disorder, which included levels of protein C, protein S, antithrombin III, lupus anticoagulant, anticardiolipin antibodies, resistance to activated protein $\mathrm{C}$ (Factor V Leiden) and a complete blood count, revealed no abnormality. Screening for occult malignancies with fecal occult blood, thoracic and abdominal CT scans and GI series was negative.

Two weeks before admission, edema in the right leg worsened and there was associated local heat and redness. The patient did not experience any fever, chills, night sweats, palpitations, sleep disturbances, urinary or bowel abnormalities, joint complaints, or neurological deficits.

The patient had a history of epilepsy in childhood, controlled with carbamazepine. Her past medical history also included a subtotal hysterectomy seven years before admission, cholecystectomy six years before, surgery for renal calculi two years before, an episode of bacterial pneumonia two years before, and a left carpal tunnel syndrome in the past year, which had resolved.

History of miscarriage, oral contraceptive use, smoking, alcohol abuse, illicit drugs, or risk factors for HIV infection was negative. She was a very active housewife before her current illness. Family history was negative.

On admission, the patient was afebrile and in no acute distress. Heart rate was $110 \mathrm{bpm}$, respirations were 23 per minute and blood pressure was 130/90 $\mathrm{mm} \mathrm{Hg}$. Examination of the head and neck revealed no lymphadenopathy and a thyroid gland of normal size and consistency.

On cardiovascular exam, the jugular venous pressure was distended to the angle of the jaw at $45^{\circ}$ with a large $\mathrm{V}$ wave and a positive 
hepatojugular reflux. Carotid pulses were normal; no bruits were heard.

A right ventricular heave was palpated at the left sternal border and the pulmonary component of the second heart sound was felt. There was a third heart sound at the lower left sternal border, and the pulmonic component of the second heart sound was increased. A holosystolic crescendo murmur of III/VI intensity that increased during inspiration was heard at the lower left sternal border.

The patient was using accessory muscles for respiration. The lungs were clear. Abdominal examination revealed moderate ascites, extensive collateral circulation of the inferior vena cava type and a tender liver $6 \mathrm{~cm}$ below the costal margin.

Examination of the extremities revealed bilateral leg edema, with signs of chronic venous insufficiency, including hyperpigmentation, superficial varicosities and shiny, thin skin. Tenderness was elicited upon compression of the calf muscles.

Laboratory investigations revealed a low hematocrit $(36 \%)$ and hemoglobin $(11 \mathrm{~g} / \mathrm{dl})$ with normal platelet and white cell counts. Electrolytes and renal function tests were unremarkable. Arterial blood gases were as follows: $\mathrm{pH}, 7.39 ; \mathrm{pCO}_{2}, 30 \mathrm{mmHG} \mathrm{pO}_{2}, 73$ mmHG; $\mathrm{O}_{2}$ Sat., $94 \% ; \mathrm{HCO}_{3}, 18 \mathrm{meq} / \mathrm{L}$. These results suggested that the symptoms of fatigue and dyspnea could not be explained by a either a severe anemia or by decreased blood oxygen content which characterize hematological abnormalities and severe pulmonary parenchymal disorders, respectively.

A chest X-ray revealed right heart enlargement and signs of pulmonary arterial hypertension, with enhanced hilar shadows and decreased peripheral pulmonary vasculature. Echocardiographic examination demonstrated severe pulmonary arterial hypertension (estimated pulmonary systolic pressure of $70 \mathrm{mmHg}$, with a leftward bulging of the interventricular septum), moderate tricuspid regurgitation, and right atrial and ventricular enlargement, with normal left ventricular function. No left to right shunts were detected. This suggested the presence of a pulmonary vascular disease as opposed to a cardiac cause to explain the pulmonary hypertension.

A ventilation-perfusion scan revealed areas of perfusion deficit characteristic of a pulmonary thromboembolism in the right lower lobe and in the left upper lobe, in the presence of normal ventilation (i.e. dead space), ruling out primary pulmonary hypertension (PPH) as the etiology. A duplex scan of the lower extremities showed bilateral venous thrombosis in the iliac, femoral and popliteal vessels. Some of these demonstrated signs of organization, suggesting the presence of an old thrombotic process mixed with ongoing deep venous thrombosis.

Venous dissection for right heart catheterization demonstrated multiple thrombi in the arm veins. Right heart catheterization revealed pulmonary artery and right ventricular systolic pressures of $75 \mathrm{~mm} \mathrm{Hg}$ with a mean pressure of $35 \mathrm{~mm} \mathrm{Hg}$. Pulmonary angiography demonstrated an occlusive thrombus in the right inferior lobar artery and multiple proximal thrombotic stenoses of the pulmonary vessels (Fig $\underline{\text { s. 1a and } 1 \mathrm{~b}}$ ). These results confirmed the diagnosis of severe pulmonary arterial hypertension due to chronic pulmonary thromboembolism. The proximal location of the thrombi and the relative sparing of the distal pulmonary tree rendered the lesion suitable for surgical therapy with pulmonary thromboendarterectomy. Occlusion of the inferior vena cava, as showed by cavography, precluded the insertion of an IVC filter.

A median sternotomy enabled a pulmonary thromboendartectomy to be performed. A severely hypertrophic right ventricle and multiple pulmonary thrombi in both proximal vessels were revealed; most pulmonary thrombi were covered by an intimal proliferation, characteristic of a chronic organizing process. There were no complications in the postoperative period except for moderate hypoxemia. Warfarin was prescribed and the patient was subsequently discharged after three weeks of hospitalization. A repeat pulmonary angiography three days after surgery had revealed no significant changes in the peripheral pulmonary circulation and the pulmonary artery systolic pressure was $60 \mathrm{mmHg}$. Perfusion of the right lower lobe showed only moderate improvement (Figs. 2a and $2 \mathrm{~b})$. These results were interpreted as a partial failure of recanalization, which could be due to unsuspected distal pulmonary vessel disease (1) or to rethrombosis of the pulmonary circulation. Another angiogram was planned for three months after surgery.

\section{Clinical Diagnosis:}

Hypercoagulable Syndrome of Undetermined Etiology

Chronic Thromboembolic Pulmonary Hypertension (CTPH)

\section{DISCUSSION}

Chronic pulmonary thromboembolism is a rare sequelae of acute pulmonary embolism, occurring in 0.01 to $0.2 \%$ of the estimated 600,000 yearly cases of acute pulmonary embolism in the United States $(2,3)$. It is one of the few treatable causes of pulmonary hypertension, as surgical pulmonary thromboendarterectomy is now performed in multiple specialized centers worldwide (4-6).

Untreated, pulmonary hypertension carries a poor prognosis, with a five year mortality of $70 \%$ when the mean pulmonary artery pressure is over $30 \mathrm{mmHg}$ and $90 \%$ when over $50 \mathrm{mmHg}$ (3). Clearly, more effective treatments are needed to improve the natural history of this serious disease. Until recently, the only treatment options available were anticoagulant therapy and insertion of an IVC 
filter. These measures are not curative but prophylactic, decreasing the incidence of new embolic episodes. With recent surgical advances and improvements in myocardial protection techniques, pulmonary thromboendarterectomy has become the treament of choice in selected cases.

In patients with CTPH, a history of deep venous thrombosis or pulmonary embolism is rarely detected. Usually, the onset is insidious, with dyspnea being the most frequent symptom (3). In the majority of patients, no clue as to the thromboembolic etiology of the pulmonary hypertension is elicited by history and physical examination, necessitating a high level of suspicion and specialized imaging procedures in order to make the diagnosis. This is of great importance, as the main differential diagnosis, namely primary pulmonary hypertension, left to right shunts and reactive pulmonary hypertension due to left ventricular dysfunction or valvular heart disease, have different prognosis and treatments. In the present case, the patient presented with a typical hypercoagulablity syndrome which rapidly lead to a diagnosis. This kind of presentation is, however, uncommon. A hypercoagulable state can be diagnosed in less than $10 \%$ of cases of CPTH (3), although recent series report hypercoagulability in up to $30 \%$ of cases (7). These differences are probably due to the limited number of patients studied as well as to differences in the methods used to investigate the hypercoagulable state $(8,9)$.

CTPH rarely leads to pulmonary infarction, as the hypertrophied and dilated bronchial arteries receive collateral circulation from the pulmonary arteries. Therefore, lung tissues typically remain viable even in the context of total chronic occlusion, but surgical intervention to restore the patency of the pulmonary arteries offers the potential for considerable clinical benefit.

Diagnosis requires a high degree of clinical suspicion and is based on scintigraphic and pulmonary arteriographic findings. On ventilation-perfusion scans, CTPH can be differentiated from PPH by the segmental or larger distribution of perfusion defects on ventilation-perfusion scan in CTPH as compared with the diffuse or less than segmental perfusion defects seen in PPH.

Pulmonary angiography is the gold standard for diagnosis (10) and is also necessary for assessment of the feasibility of pulmonary thromboendarterectomy. This invasive evaluation carries a mortality of $0.2 \%$ and a complication rate of $4.5 \%$ (3). Pulmonary angiography in CTPH typically shows webs, bands, stenoses and total occlusions of the proximal pulmonary tree, with intimal irregularities and rounded terminations of pulmonary branches. It must be emphasized that the interpretation of this investigation is quite different from that in acute pulmonary embolism, where a thrombus can be readily detected as a sharp filling defect in a branch of the pulmonary tree. By contrast, in CTPH, oddly shaped pulmonary arteries are frequently seen, and thrombi are rarely detected as such $(5,10)$. This atypical morphology underscores the need for an experienced angiographer.

Recently, spiral CT scanning has allowed excellent opacification of the pulmonary arteries, and thrombi in the pulmonary arteries can be directly visualized (11). This non-invasive method is being evaluated as an alternative to pulmonary angiography. CT depiction of bronchial arteries, dilatation and tortuosity provides indicators for CTPH, even though its severity cannot be estimated (12). The sensitivity of spiral CT scans in confirming the diagnosis of CTPH is greater than $90 \%$, and leads to a definitive decision regarding the operability of the patient in more than $80 \%$ of cases (13). This imaging method holds promise in the diagnosis, evaluation of operative feasibility and postoperative follow up of CTPH patients, but at present, pulmonary angiography remains the gold standard.

Pulmonary thromboendarterectomy is now the treatment of choice for $\mathrm{CPTH}$, providing a potential cure for an otherwise fatal disease. Performed at a limited number of centers, it is a complex procedure involving a median sternotomy with cardiopulmonary bypass and deep hypothermia, necessitating long periods of total cardiac arrest (2). Current criteria for surgery include: NYHA class III and IV; pulmonary vascular resistance (PVR) greater than 300 dynes $/ \mathrm{s} \mathrm{cm}^{-5}$ (7); thrombus location in the main, lobar, or segmental arteries; absence of severe associated disease; and patient willingness to accept a surgical mortality of 10 to $20 \%$. CTPH is defined in current trials as a mean resting pulmonary artery pressure greater than $25 \mathrm{mmHg}$ with a mean pulmonary wedge pressure less than $12 \mathrm{mmHg}$, associated with at least one segmental or larger defect on ventilation-perfusion scan and accompanied by an angiographic pattern of CTPH as described above (7).

Given the relatively high mortality of the procedure, which has been reported in experienced centers to be in the range of $8.7 \%$ (2) to $25 \%$ (14), and its potentially serious complications (specifically persistent pulmonary hypertension by means of rethrombosis or incomplete thromboendarterectomy, and pulmonary reperfusion edema) $(5,15)$, careful selection of patients and operative variables are crucial in achieving good results. Recent trials have attempted to define predictors of operative mortality $(3,14)$, of which the most important are severe hemodynamic disease (PVR greater than 1100 dynes $/ \mathrm{s} / \mathrm{cm}^{-5}(7)$, pulmonary artery systolic pressure greater than $50 \mathrm{~mm} \mathrm{Hg}$ ), reduction in PVR of less than $50 \%$ after surgery, and prolonged bypass time.

The reported results of pulmonary thromboendarterectomy are very encouraging $(3-6,16)$. Heart failure in most patients improves to NYHA class I or II, with significant reductions in PVR and right ventricular dimensions, and improvements in cardiac index and right ventricular function. Most importantly, these patients tend to improve markedly in functional status, returning to a normal productive life. Ninety-five percent of patients are in NYHA class I or II at 12 months (17), and sustained, progressive improvement has been observed after patients have been followed for approximately 2 years $(16,18)$. It must be emphasized as well that the only alternative intervention in these severely ill patients is lung or heart-lung transplantation, with attendant problems of organ donation and immunosuppression. The rejection and one year survival rate is approximately $70 \%$ while the four year survival rate is only $40 \%$ (19). 
The most important aspect of long term postoperative care is that patients need to be maintained on lifelong anticoagulation therapy, in addition to the insertion of an IVC filter, which is usually performed preoperatively in the absence of contraindications.

Although no marked clinical improvement was evident in the patient presented here at the time of this report, this case underscores the fact that pulmonary thromboendarterectomy is a relatively safe, feasible and a potentially curative treatment for CTPH. In this particular case, management will include repeating non-invasive and angiographic evaluation regularly, to document possible progressive improvements. Meanwhile, the patient is receiving intensive anticoagulant therapy and is starting on a rehabilitation protocol.

\section{ACKNOWLEDGMENT}

The authors are indebted to Prof. Edson A. Saad for helpful commentary on the manuscript.

\section{REFERENCES}

1. Hirsch AM, Moser KM, Auger WR, et al. Unilateral pulmonary artery thrombotic occlusion: is distal arteriopathy a consequence? American Journal of Respiratory and Critical Care Medicine 154(2 Pt 1): 491-496; 1996.

2. Jamieson SW, Auger WR, Fedullo PF. Experience and results with 150 pulmonary thromboendarterectomy operations over a 29 month period. Journal of Thoracic and Cardiovascular Surgery 106(1): 116-127; 1993.

3. Viner SM, Bagg BR, Auger WR, et al. The management of pulmonary hypertension secondary to chronic thromboembolic disease. Progress in Cardiovascular Diseases 37(2): 79-92; 1994.

4. Fedullo PF, Auger WR, Moser KN, et al. Chronic thromboembolic pulmonary hypertension. Clinics in Chest Medicine 16(2): 353$374 ; 1995$.

5. Iversen S. Surgical treatment of thromboembolism-induced pulmonary hypertension. Zeitschrift fur Kardiologie 83(Suppl 6): 193$199 ; 1994$.

6. Klepetko W, Moritz A, Burghuber OC, et al. Chronic thromboembolic pulmonary hypertension and its treatment with pulmonary thromboendarterectomy. Wiener Klinische Wochenschrift 107(13): 396-402; 1995.

7. Simonneau G, Brenot F, Musset D, et al. Surgical management of unresolved pulmonary embolism. A personal series of 72 patients. Chest 107(1 Suppl): 52S-55S; 1995.

8. Sandoval J, Amigo MC, Barragan R, et al. Primary antiphospholipid syndrome presenting as chronic thromboembolic pulmonary hypertension. Treatment with thromboendarterectomy. Journal of Rheumatology 23(4): 772-775; 1996.

9. Cucurull E, Ordi R, Murtra M, et al. Pulmonary thromboendarterectomy in a patient with primary antiphospholipid syndrome. Medicina Clinica (Barcelona) 106(13): 498-500; 1996.

10. Greenspan RH. Pulmonary Angiography and the diagnosis of pulmonary embolism. Progress in Cardiovascular Diseases 37(2): 93-105; 1994.

11. Kontrus M, Bankier AA, Fleishmann D, et al. Spiral CT in chronic lung thromboembolism. Radiologe 36(6): 496-502; 1996.

12. Kauczor HU, Schwickert HC, Mayer E, et al. Spiral CT of bronchial arteries in chronic thromboembolism. Journal of Computer Assisted Tomography 18(6): 855-861; 1994.

13. Schwickert HC, Schweden F, Schild, HH, et al. Pulmonary arteries and lung parenchyma in chronic pulmonary embolism: preoperative and postoperative CT findings. Radiology 191(2): 351-357; 1994.

14. Hartz RS, Byrne JG, Levitsky S, et al. Predictors of mortality in pulmonary thromboendarterectomy. Annals of Thoracic Surgery 62(5):1255-1259; 1996.

15. Levinson RM, Shure D, Moser KM. Reperfusion pulmonary edema following pulmonary thromboendarterectomy. American Review of Respiratory Disease 134(6): 1241-1245; 1986.

16. Mayer E, Dahm M, Hake U, et al. Mid-term results of pulmonary thromboendarterectomy for chronic thromboembolic pulmonary hypertension. Annals of Thoracic Surgery 61(6):1788-1792; 1996.

17. Moser KM, Auger WR, Fedullo PF. Chronic thromboembolic pulmonary hypertension: clinical picture and surgical treatment. 
European Respiratory Journal 5(3): 334-342; 1992.

18. Mayer E, Dahm M, Hake U, et al. Thromboendarterectomy in chronic thromboembolic pulmonary hypertension. hemodynamics and right-heart function over the long term. Deutsche Medizinische Wochenschrift 121(1-2): 9-15; 1996.

19. Chapelier A, Vouhe P, Macchiarini P et al. Comparative outcome of heart-lung and lung transplantation for pulmonary hypertension: report of 30 cases. Journal of Thoracic and Cardiovascular Surgery 106(2): 299-307; 1993.

\section{BIOGRAPHY}

Eduardo B. Saad is currently a sixth year medical student at the Universidade Federal do Rio de Janeiro (Federal University of Rio de Janeiro) (Rio de Janeiro, RJ, Brazil). His research interests include cardiovascular medicine and vascular disorders and his future specialty interests are in cardiology, cardiac electrophysiology, and intensive care medicine.

Carlos E. Reis is currently a sixth year medical student at the Universidade Federal do Rio de Janeiro (Federal University of Rio de Janeiro) (Rio de Janeiro, RJ, Brazil). His research interests are in neuroscience. He plans to pursue a specialty in neurology following graduation.

Cynthia F. Pontes, M.D., is currently a second year pulmonary medicine resident at the Universidade Federal do Rio de Janeiro. Her research interests are in pulmonary physiology and pathophysiology. Her future specialty interests are in pulmonary medicine, bronchoscopy, mechanical ventilation and intensive care medicine.

Copyright (C) 1997 by MJM 